\title{
High temperature rapid thermal annealing of phosphorous ion implanted InAs/InP quantum dots
}

\author{
S. Barik, ${ }^{a)}$ H. H. Tan, and C. Jagadish \\ Department of Electronic Materials Engineering, Research School of Physical Sciences and Engineering, \\ The Australian National University, Canberra, Australian Capital Territory 0200, Australia
}

(Received 15 January 2007; accepted 26 January 2007; published online 27 February 2007)

\begin{abstract}
The effect of high temperature annealing of the InAs/InP quantum dots (QDs) containing a thin GaAs interlayer is investigated. The QDs are rapid thermally annealed at 750, 800, 850, and $900{ }^{\circ} \mathrm{C}$ for $30 \mathrm{~s}$. The QDs with the GaAs interlayer show good thermal stability up to $850{ }^{\circ} \mathrm{C}$ as well as enhanced integrated photoluminescence (PL) intensity and reduced PL linewidth. The effect of high energy $(450 \mathrm{keV})$ phosphorous ion implantation at room temperature with doses of $5 \times 10^{11}-5$ $\times 10^{13}$ ions $/ \mathrm{cm}^{2}$ with subsequent high temperature $\left(750-850^{\circ} \mathrm{C}\right)$ rapid thermal annealing is also studied. A large implantation-induced energy shift of up to $309 \mathrm{meV}(400 \mathrm{~nm})$ is observed. The implanted samples annealed at $850^{\circ} \mathrm{C}$ show reduced PL linewidth and enhanced integrated PL intensity compared to the implanted samples annealed at $750{ }^{\circ} \mathrm{C}$. (C) 2007 American Institute of Physics. [DOI: 10.1063/1.2710006]
\end{abstract}

InAs quantum dots (QDs) grown on InP substrates are promising active materials for high performance optoelectronic devices operating at long wavelength region $(1.3-2 \mu \mathrm{m})$ and suitable for optical fiber communications. The self-assembled QDs grown by the Stranski-Krastanov growth mode have large fluctuations in size, shape, strain, and composition leading to inhomogeneous broadening of the photoluminescence (PL) emission. But narrower PL emission is required for device applications. Narrower PL linewidth as well as blueshift of the PL emission was reported in rapid thermally annealed InGaAs/GaAs QDs (Ref. 1) and InAs/GaAs QDs. ${ }^{2}$ Enhancements in the integrated PL intensity were also observed in the annealed InAs/GaAs QDs covered with a $\mathrm{SiO}_{2}$ film. ${ }^{2,3}$ However, Babiński et al. ${ }^{4}$ reported the disappearance of InAs/GaAs QDs at a temperature of about $800^{\circ} \mathrm{C}$ due to strain-enhanced lateral $\mathrm{In} / \mathrm{Ga}$ interdiffusion. InAs QDs suffer 7.2\% lattice mismatch on GaAs substrates. InAs QDs have less lattice mismatch of $3.2 \%$ on InP substrates resulting in relatively large InAs QDs with a thin wetting layer $[\sim 1.5 \mathrm{ML}]$ (Ref. 5). Chia et al. ${ }^{6}$ performed rapid thermal annealing of InAs/InP QDs from 600 to $900{ }^{\circ} \mathrm{C}$ and did not observe any disappearance of the QDs. However, they reported a large thermal energy shift (more than $325 \mathrm{meV}$ ) due to annealing of the InAs/InP QDs at $900{ }^{\circ} \mathrm{C}$. Girard et $\mathrm{al}^{7}{ }^{7}$ performed thermal annealing of InAs/InP QDs at $650-800{ }^{\circ} \mathrm{C}$ for $210 \mathrm{~s}$ and reported an increase in the PL energy up to $120 \mathrm{meV}$ revealing poor thermal stability of the InAs/InP QDs. None of these groups grew InAs/InP QDs containing thin GaAs interlayers but a thin GaAs interlayer has been reported to improve the optical and structural qualities of InAs/InP QDs. ${ }^{8,9}$ In this letter, we present the results of rapid annealing of the InAs/InP QDs containing a thin GaAs interlayer at temperatures ranging from 750 to $900{ }^{\circ} \mathrm{C}$ for $30 \mathrm{~s}$. Our results show that the GaAs interlayer improves the thermal stability of the InAs/InP QDs. Our results also show that high temperature annealing reduces the PL linewidth and enhances the integrated PL intensity and thereby improves the uniformity and the optical quality of the InAs/InP QDs.

\footnotetext{
${ }^{\text {a)} E l e c t r o n i c ~ m a i l: ~ s n b 109 @ r s p h y s s e . a n u . e d u . a u ~}$
}

To monolithically integrate active/passive components of optoelectronic devices, selective and controllable spatial band gap tuning is necessary. Post growth QD intermixing can increase the band gap energy and reduce the PL linewidth. Ion implantation-induced QD intermixing has many advantages, such as compatibility with planar processing and reproducibility. The degree and depth of intermixing can be selectively controlled by choosing right implant conditions such as ion dose, ion energy, implantation temperature, and mask profile. In this letter, we also report phosphorous ion implantation at room temperature directly over the QD region followed by high temperature $\left(750-850^{\circ} \mathrm{C}\right)$ rapid thermal annealing of the samples. We observe a large implantation-induced energy shift of up to $309 \mathrm{meV}$ and show that high temperature annealing does not reduce differential band gap energy shift significantly owing to good thermal stability of the QDs. Our results also show that high temperature annealing improves the integrated PL intensity and reduces the PL linewidth of the $\mathrm{P}$ ion implanted InAs/InP QDs.

InAs QDs are grown on semi-insulating (100) InP substrates using horizontal flow metal-organic chemical vapor deposition at a pressure of 180 mbar. Trimethylindium, trimethylgallium, $\mathrm{PH}_{3}$, and $\mathrm{AsH}_{3}$ are used as precursors, and $\mathrm{H}_{2}$ as the carrier gas. First a $700 \mathrm{~nm}$ InP layer is grown at $650{ }^{\circ} \mathrm{C}$. To minimize the As/P exchange reaction a $0.6 \mathrm{~nm}$ thick GaAs interlayer is also grown at $650{ }^{\circ} \mathrm{C}$ before the growth of a QD layer. The growth temperature is then reduced to $520{ }^{\circ} \mathrm{C}$ and the InAs QDs are deposited for $6.5 \mathrm{~s}$ at the rate of $0.16 \mathrm{~nm} / \mathrm{s}$. The QDs are immediately capped without any growth interruption with a $300 \mathrm{~nm} \mathrm{InP}$ layer while the temperature is increased to $650{ }^{\circ} \mathrm{C}$. Atomic force microscopy on uncapped QDs and plan-view transmission electron microscopy on capped QDs are carried out to measure the height and size of the QDs. The QDs have a mean height of $8.2 \mathrm{~nm}$ and a mean diameter of $25 \mathrm{~nm}$.

The QD samples are implanted at room temperature with $\mathrm{P}$ ions with doses of $5 \times 10^{11}-5 \times 10^{13}$ ions $/ \mathrm{cm}^{2}$. The energy of the ions is $450 \mathrm{keV}$ which places the damage peak directly over the QD region. The implantation is carried out at $7^{\circ}$ off the $\langle 100\rangle$ direction to minimize channeling effects. The un- 

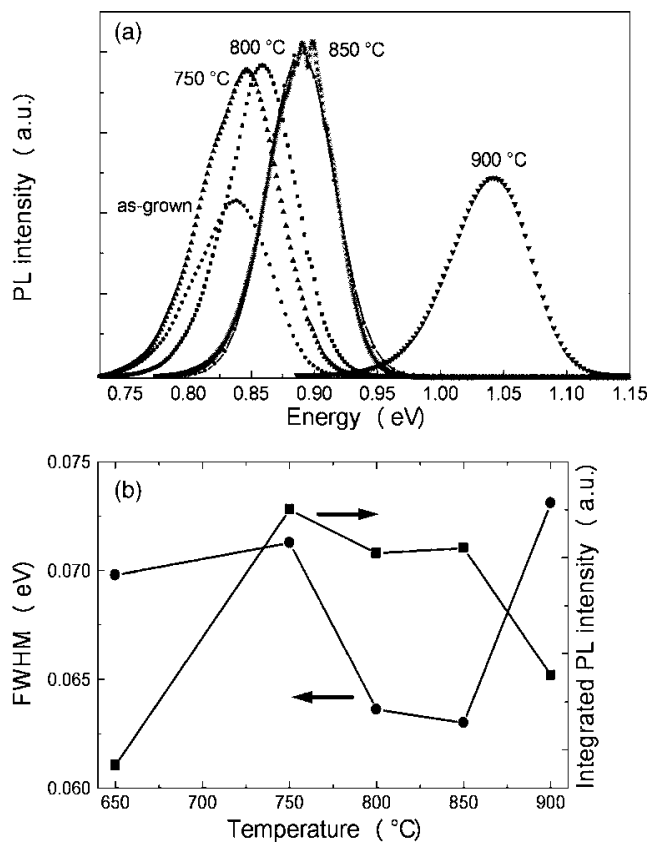

FIG. 1. (a) Photoluminescence (PL) spectra measured at $77 \mathrm{~K}$ of the asgrown and the as-grown and annealed InAs/InP quantum dots (QDs). The QDs are annealed at $750,800,850$, and $900{ }^{\circ} \mathrm{C}$ for $30 \mathrm{~s}$. A Gaussian fit of the PL spectrum of the QDs annealed at $850^{\circ} \mathrm{C}$ is shown by the line with closed circles. (b) The full widths at half maximums (FWHMs) of the PL spectra and the integrated PL intensities of the QDs annealed at different temperatures. The data for the as-grown QDs are plotted for $650{ }^{\circ} \mathrm{C}$ due to their thermal treatment at this temperature during growth.

implanted as-grown samples are annealed for $30 \mathrm{~s}$ at 750 , 800,850 , and $900{ }^{\circ} \mathrm{C}$ in argon ambient using a rapid thermal processor. The implanted samples are annealed at either 750 or $850{ }^{\circ} \mathrm{C}$ for $30 \mathrm{~s}$ to study the effects of annealing temperature on the implanted samples. To protect the top InP layer from the excessive loss of $\mathrm{P}$ atoms during annealing, the samples are sandwiched between two fresh pieces of semiinsulating InP substrates. Photoluminescence (PL) measurements are carried out at $77 \mathrm{~K}$ using the $532 \mathrm{~nm}$ line of a frequency doubled diode pumped solid state laser. The PL signal is collected by a thermoelectrically cooled InGaAs photodetector with a built-in preamplifier after dispersing through a $0.5 \mathrm{~m}$ monochromator.

The PL spectra of the as-grown sample and the as-grown samples annealed at $750,800,850$ and $900{ }^{\circ} \mathrm{C}$ for $30 \mathrm{~s}$ are shown in Fig. 1(a). The PL spectrum of the sample annealed at $850{ }^{\circ} \mathrm{C}$ has a dip at $0.895 \mathrm{eV}$ due to atmospheric absorption. A Gaussian fit of this spectrum is shown by the line with closed circles and used to calculate the PL peak energy and the full width at half maximum (FWHM). The thermal energy shift for annealing at temperatures less than $800{ }^{\circ} \mathrm{C}$ is very small. Salem et al..$^{5}$ reported no significant energy shift in their InAs/InP quantum stick samples annealed at $650{ }^{\circ} \mathrm{C}$ but reported a large energy shift for annealing temperatures higher than $700{ }^{\circ} \mathrm{C}$. In our case, the thermal energy shift for the QD samples annealed at $850^{\circ} \mathrm{C}$ is $54 \mathrm{meV}$, much smaller than the energy shift observed by Chia et al. in the InAs/InP QDs annealed at the same temperature. ${ }^{6}$ Chia et al. grew InAs QDs directly on InP buffers without growing any GaAs interlayer between the QD layer and the InP buffer layer. They observed prominent increase in the thermal energy shift as annealing temperature increased up to $900{ }^{\circ} \mathrm{C}$. In our case, the thermal energy shift for the QD sample anDownloaded 24 Feb 2008 to 150.203.178.41. Redistribution subject

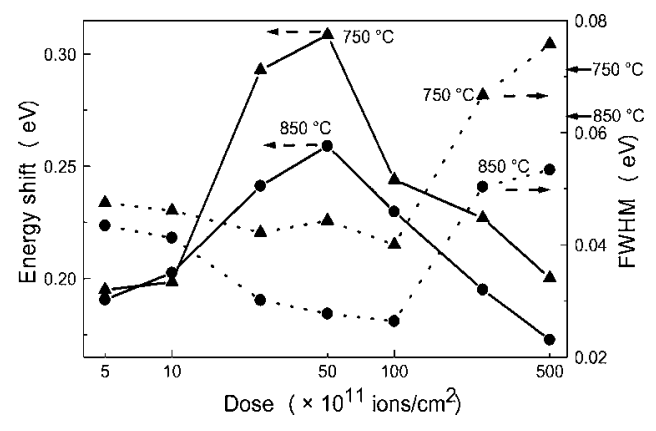

FIG. 2. The implantation-induced energy shift and the full width at half maximum (FWHM) of the photoluminescence (PL) spectrum of the InAs/InP quantum dots (QDs) implanted with various $\mathrm{P}$ ion doses. The FWHMs of the PL spectra of the unimplanted and annealed QDs are also shown (by solid arrows) for comparison. All samples are annealed at either 750 or $850^{\circ} \mathrm{C}$ for $30 \mathrm{~s}$.

nealed at $900{ }^{\circ} \mathrm{C}$ is quite large $(204 \mathrm{meV})$ but it is less than the thermal energy shift (more than $325 \mathrm{meV}$ ) of the similar samples grown by Chia et al. We believe that a thin GaAs interlayer minimizes the As/P exchange reaction and improves the thermal stability of the InAs/InP QDs. GaAs is under tensile strain due to $3.7 \%$ lattice mismatch on InP substrates and can partially compensate the compressive strain of the InAs QDs. It should reduce the strain gradient for interdiffusion leading to better thermal stability. Similar effect was observed in the InGaAs/GaAs QDs grown with $\mathrm{GaP}$ strain compensation layers and was attributed to the partial compensation of the strain in the QDs by the tensilestrained GaP layer. ${ }^{10}$

The large thermal energy shift observed in our samples at $900{ }^{\circ} \mathrm{C}$ is due to material degradation. We have observed a rough surface after annealing at $900{ }^{\circ} \mathrm{C}$ due to $\mathrm{P}$ desorption from the InP cap layer. This $\mathrm{P}$ outdiffusion creates defects leading to strong intermixing in the QDs. As a result of material degradation, the integrated PL intensity, decreases and the FWHM increases as shown in Fig. 1(b). But the samples annealed at temperatures lower than $900{ }^{\circ} \mathrm{C}$ show much enhanced integrated PL intensity compared to the as-grown sample, as shown in Fig. 1(b). Similar increase in the integrated PL intensity was observed in annealed InAs/InP QDs ${ }^{6}$ and InAs/InP quantum sticks. ${ }^{5}$ Rapid thermal annealing may decrease nonradiative recombination centers leading to an increase in the PL intensity. Since growth temperature rises to $650^{\circ} \mathrm{C}$ during the InP cap layer growth, the FWHM and the integrated PL intensity for the as-grown sample are plotted for $650{ }^{\circ} \mathrm{C}$ in Fig. 1(b). In addition to the increase in the integrated PL intensity, the FWHM decreases to 64 and $63 \mathrm{meV}$ for the samples annealed at 800 and $850^{\circ} \mathrm{C}$, respectively, from the FWHM of $70 \mathrm{meV}$ for the as-grown sample. It shows that high temperature annealing also decreases the overall variation in QD size, strain, and composition profile.

Figure 2 shows the implantation-induced energy shifts of the QDs annealed at either 750 or $850^{\circ} \mathrm{C}$ or for various implantation doses. The implantation-induced energy shift is defined as the difference between the PL energy peaks of the implanted and as-grown (unimplanted) samples which are annealed at the same temperature. The implantation-induced energy shift increases with increasing implantation dose up to the dose of $5 \times 10^{12}$ ions $/ \mathrm{cm}^{2}$ irrespective of annealing temperature. The implantation-induced energy shift for the QD sample implanted with a dose of $5 \times 10^{12} \mathrm{ions} / \mathrm{cm}^{2}$ and
to AIP license or copyright; see http://apl.aip.org/apl/copyright.jsp 


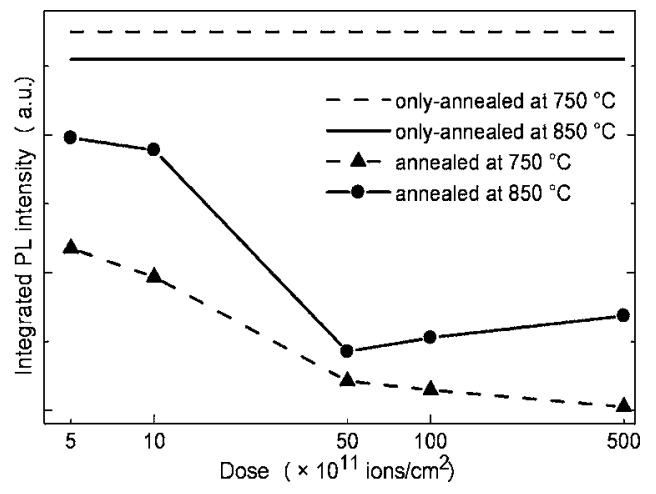

FIG. 3. The integrated photoluminescence (PL) intensity of the InAs/InP quantum dots (QDs) implanted with various $\mathrm{P}$ ion doses. The integrated PL intensities of the unimplanted and annealed samples (only annealed) are also shown for comparison. The QDs are annealed at either 750 or $850{ }^{\circ} \mathrm{C}$ for $30 \mathrm{~s}$.

annealed at $750{ }^{\circ} \mathrm{C}$ for $30 \mathrm{~s}$ is $309 \mathrm{meV}(400 \mathrm{~nm})$. For the doses more than $5 \times 10^{12}$ ions $/ \mathrm{cm}^{2}$ the implantation-induced energy shift starts to decrease from the maximum value. As the implantation dose increases, more complex defect clusters start to form. As a result the implantation-induced energy shift reduces due to the reduction in the availability of point defects which cause intermixing. The thermal energy shift of the sample annealed at $850{ }^{\circ} \mathrm{C}$ is $46 \mathrm{meV}$ more than that of the sample annealed at $750^{\circ} \mathrm{C}$ as can be seen in Fig. 1(a). Hence the implanted samples annealed at $850{ }^{\circ} \mathrm{C}$ show reduced implantation-induced energy shift compared to the implanted samples annealed at $750{ }^{\circ} \mathrm{C}$. However, for low ion doses (less than $10^{12}$ ions $/ \mathrm{cm}^{2}$ ), the implantation-induced energy shifts in the samples annealed at $850{ }^{\circ} \mathrm{C}$ are similar to the shifts in the samples annealed at $750{ }^{\circ} \mathrm{C}$. This shows that although the higher annealing temperature causes larger thermal energy shift, high temperature annealing initiates more implantation-induced intermixing when the implantation dose is low.

The FWHM of the samples implanted with various doses and annealed at either 750 or $850{ }^{\circ} \mathrm{C}$ are also shown in Fig. 2. The values of the FWHM of the unimplanted QDs annealed at either 750 or $850{ }^{\circ} \mathrm{C}$ are shown (by solid arrows) for comparison. It is interesting to note that for low doses the FWHM for the implanted and annealed sample decreases noticeably compared to that of the annealed but unimplanted QD sample. Ion implantation-induced intermixing reduces the overall variation in QD size, shape, strain, and composition profiles of the QDs. The FWHM continues to decrease up to the implantation dose of $1 \times 10^{13}$ ions $/ \mathrm{cm}^{2}$, but for higher doses the FWHM increases. As noted earlier, at higher doses more residual defects remain in the samples even after annealing leading to nonuniform intermixing. Similar effect was observed by Salem et al. in InAs/InP quantum stick samples. ${ }^{5}$ The FWHM for the sample annealed at $850{ }^{\circ} \mathrm{C}$ is less than that for the sample annealed at $750{ }^{\circ} \mathrm{C}$ for each dose. As noted earlier, high temperature annealing initiates more intermixing and thereby improves the uniformity in shape, size, strain, and composition of the QDs leading to further reduction in the FWHM.

In Fig. 3, we compare the integrated PL intensity of the QD samples implanted with various doses and annealed at either 750 or $850{ }^{\circ} \mathrm{C}$. Compared to the as-grown and annealed (unimplanted) samples, the implanted samples annealed at $750{ }^{\circ} \mathrm{C}$ show continuous decrease in the integrated Downloaded 24 Feb 2008 to 150.203.178.41. Redistribution subject
PL intensity as the implantation dose increases. The PL intensity does not recover well due to insufficient removal of nonradiative defects as the implantation dose increases. High temperature annealing is required to remove these defects more efficiently. As shown in Fig. 3, although the integrated PL intensity for the as-grown (unimplanted) sample annealed at $850^{\circ} \mathrm{C}$ is less than that of the as-grown (unimplanted) sample annealed at $750{ }^{\circ} \mathrm{C}$, the integrated PL intensities for the implanted samples annealed at $850{ }^{\circ} \mathrm{C}$ are greater than those of the implanted samples annealed at $750^{\circ} \mathrm{C}$ for all implantation doses. In general, the PL intensity recovery is not good for doses higher than $5 \times 10^{12} \mathrm{ion} / \mathrm{cm}^{2}$. As pointed out earlier, defects are created directly into the QD layer due to the ion energy used in this experiment. For higher doses, too many defects are created into the QD layer and annealing even at high temperature cannot remove them completely. If the damage peak is not created into the QD layer, the PL intensity recovery could be better. However, the damage peak created too far away from the QD layer may result in very little or no intermixing.

In conclusion, we have studied the effects of high temperature annealing of the InAs/InP QDs containing a thin GaAs interlayer. These QDs show good thermal stability up to $850{ }^{\circ} \mathrm{C}$. The rapid thermally annealed QD samples show enhanced integrated PL intensity. The QD samples annealed at high temperatures $\left(800\right.$ and $850{ }^{\circ} \mathrm{C}$ ) show reduced FWHM which is attractive for QD based optoelectronic devices. We have also studied the effects of high temperature annealing on the $\mathrm{P}$ ion implantation-induced intermixing of the InAs/InP QDs. The QD sample implanted with 5 $\times 10^{12}$ ions $/ \mathrm{cm}^{2}$ and annealed at $750{ }^{\circ} \mathrm{C}$ shows the implantation-induced energy shift of up to $309 \mathrm{meV}$ $(400 \mathrm{~nm})$. High temperature annealing does not reduce the implantation-induced component of energy shift by large extent; it actually initiates additional ion implantation-induced intermixing when the implantation dose is low. The implanted samples annealed at $850{ }^{\circ} \mathrm{C}$ show reduced FWHM and enhanced integrated PL intensity compared to the implanted samples annealed at $750{ }^{\circ} \mathrm{C}$. Our results show that high temperature annealing after $\mathrm{P}$ ion implantation could be beneficial for selective band gap tuning in InAs/InP QD system paving the way to monolithically integrate QD based optoelectronic devices.

The authors gratefully acknowledge financial support from the Australian Research Council.

${ }^{1}$ R. Leon, Y. Kim, C. Jagadish, M. Gal, J. Zou, and D. J. H. Cockayne, Appl. Phys. Lett. 69, 1888 (1996).

${ }^{2}$ S. Malik, C. Roberts, R. Murray, and M. Pate, Appl. Phys. Lett. 71, 1987 (1997).

${ }^{3}$ S. J. Xu, X. C. Wang, S. J. Chua, C. H. Wang, W. J. Fan, J. Jiang, and X. G. Xie, Appl. Phys. Lett. 72, 3335 (1998).

${ }^{4}$ A. Babiński, J. Jasiński, R. Bożek, A. Szepielow, and J. M. Baranowski, Appl. Phys. Lett. 79, 2576 (2001).

${ }^{5}$ B. Salem, V. Aimez, D. Morris, A. Turala, P. Regreny, and M. Gendry, Appl. Phys. Lett. 87, 241115 (2005).

${ }^{6}$ C. K. Chia, S. J. Chua, S. Tripathy, and J. R. Dong, Appl. Phys. Lett. 86, 051905 (2005)

${ }^{7}$ J. F. Girard, C. Dion, P. Desjardins, C. N. Allen, P. J. Poole, and S. Raymond, Appl. Phys. Lett. 84, 3382 (2004).

${ }^{8}$ Q. Gong, R. Nötzel, P. J. v. Veldhoven, T. J. Eijkemans, and J. H. Wolter, Appl. Phys. Lett. 84, 275 (2004).

${ }^{9}$ S. Barik, H. H. Tan, C. Jagadish, N. Vukmirović, and P. Harrison, Appl. Phys. Lett. 88, 193112 (2006).

${ }^{10}$ P. Lever, H. H. Tan, and C. Jagadish, J. Appl. Phys. 95, 5710 (2004). 